\title{
PANDEMIA Y DURACIÓN DEL DESEMPLEO JUVENIL EN CALI*
}

\author{
Jhon James Mora ${ }^{\mathrm{a}}$ \\ Diana Y. Herrera \\ Juan Fernando Alvarez'
}

* DOI: https.//doi.org/10.18601/01245996.v24n.46.10 Recepción: 27-052021, modificación final: 05-11-2021, aceptación: 29-11-2021. Sugerencia de citación: Mora, J. J., Herrera, D. Y. y Álvarez, J. F. (2022). Pandemia y duración del desempleo juvenil en Cali. Revista de Economía Institucional, 24(46), 195-216.

a Profesor titular de la Universidad Icesi, Cali, Colombia. Director, grupo de economía, políticas publicas y métodos cuantitativos. Coordinador Componente de Medición Económica, Proyecto un Valle del Conocimiento, y del Observatorio Regional del Mercado de Trabajo, [jjmora@icesi.edu.co], [https://orcid.org/0000-0002-9974-1874].

b Candidata a Doctora en Economía de los Negocios, Universidad Icesi. Grupo de investigación: Economía, Políticas Públicas y Métodos Cuantitativos, Universidad Icesi, [dyherrera@icesi.edu.co], [https://orcid.org/00000001-5110-0683]

c Doctorando en Economía de los Negocios, Universidad Icesi. Grupo de investigación: Economía, Políticas Públicas y Métodos Cuantitativos, Universidad Icesi, [juan.alvarez15@icesi.edu.co], [https://orcid.org/0000-00024290-023X]. 


\section{Pandemia y duración del desempleo juvenil en Cali}

Resumen La crisis sanitaria provocada por la pandemia de Covid-19 ha tenido graves implicaciones en la dinámica laboral, tanto a nivel nacional como local, que afectan principalmente a los jóvenes. Utilizando datos oficiales y primarios, este articulo explora el comportamiento del desempleo juvenil en la ciudad de Cali durante la pandemia. Mediante la estimación de modelos paramétricos y no paramétricos, los resultados revelan que el tiempo que tardan los jóvenes en salir del desempleo se habría duplicado, con una probabilidad menor para las mujeres jóvenes. Sugiere, entonces, la necesidad de diseñar instrumentos específicos de política pública que reconozcan esta problemática estructural.

Palabras clave: duración del desempleo, covid-19, Kaplan-Meir, jóvenes; JEL: J64, $\mathrm{C} 41, \mathrm{~J} 13$

\section{Pandemic and duration of youth unemployment in Cali}

Abstract The health crisis caused by the Covid-19 pandemic has had serious implications on labor dynamics, both nationally and locally, with young people principally being affected. Using official and primary data, this article explores the behavior of youth unemployment in the city of Cali during the pandemic. Through the estimation of parametric and non-parametric models, our results show that the time it takes young people to exit unemployment would have doubled, evidencing a lower probability of employment among young women. Finally, we suggest the need to design specific public policy instruments that recognize these structural problems.

Keywords: Unemployment duration, Covid-19, Kaplan-Meier. youth people; JEL: $\mathrm{J} 64, \mathrm{C} 41, \mathrm{~J} 13$

\section{Pandemia e duração do desemprego juvenil em Cali}

Resumo A crise de saúde causada pela pandemia Covid-19 teve graves Implicações na dinâmica do trabalho, tanto nacional quanto localmente, que afetam principalmente os jovens. Usando dados oficiais e primários, este artigo explora o comportamento do desemprego juvenil na cidade de Cali durante a pandemia. Ao estimar modelos paramétricos e não paramétricos, os resultados revelam que o tempo que os jovens levam para sair do desemprego dobrou, com menor probabilidade para as mulheres jovens. Sugere, então, a necessidade de desenhar instrumentos de política pública específicos que reconheçam esse problema estrutural.

Palavras-chave: duração do desemprego, covid-19, Kaplan-Meir, juventude; JEL: J64, C41, J13 
L

a pandemia de Covid-19 es ante todo una crisis de salud, y a medida que el gobierno ha tratado de contener la propagación del virus, se ha transformado en una crisis económica. La pandemia ha expuesto y agravado las desigualdades existentes en el mercado de trabajo, específicamente las relacionadas con el mercado laboral formal y las grandes dificultades que tienen los jóvenes ${ }^{1}$ y las mujeres para acceder a este (Mora, 2021a). Su posición desventajosa en el mundo del trabajo es una realidad reconocida y documentada en nuestro país (Arango y Ríos, 2015; Mora et al., 2017).

Con o sin pandemia, el desempleo juvenil en el país se ha agudizado. De acuerdo con Castillo y García (2019) en los últimos años los jóvenes colombianos vienen presentado tasas de desempleo que duplican la del total del país, así como la tasa más alta de desempleo juvenil con educación superior en América Latina.

A nivel local, el desempleo de los jóvenes en la ciudad de Cali aumentó casi 10 puntos porcentuales entre febrero del 2020 (prepandemia) y febrero del 2021 (postpandemia). Esto aunado con diferencias significativas en las oportunidades para los jóvenes, reflejadas por las tasas de empleo y desempleo por etnia, así como por la ampliación del periodo en que consiguen empleo, muestra un panorama social muy complejo cuya solución no da espera, como lo evidencian las movilizaciones sociales y peticiones de la ciudadanía durante el Paro Nacional iniciado a finales del mes de abril de 2021, que sintetizan las preocupaciones e inconformismos de esta población.

Este estallido social con epicentro en Cali, desatado por la reforma tributaria, escondía un problema mayor: las escasas oportunidades de los jóvenes en el mercado laboral, que empeoraron con la pandemia.

El problema se agrava al considerar el componente espacial, dadas las fuertes disparidades en las tasas de desempleo por localización geográfica que se observan en la ciudad, específicamente en la zona de ladera y el Distrito de Aguablanca, sitios históricamente reconocidos por una alta incidencia de la pobreza y donde se radicalizaron las protestas. Por tanto, la localización geográfica juega un papel importante en las dinámicas laborales al incidir en la probabilidad que tienen los individuos de salir del desempleo, es decir, es posible que los habitantes de algunas zonas de la ciudad presenten mayores problemas al momento de emplearse.

${ }^{1}$ En Colombia se consideran jóvenes a quienes tienen una edad de 14 a 28 años (Ley 1622 de 2013, Artículo 44). Para la OIT tienen una edad de 15 a 24 años. 
La principal contribución de este artículo consiste en examinar el comportamiento del desempleo juvenil en la ciudad de Cali durante la pandemia, encontrándose que la emergencia sanitaria de la Covid-19 habría duplicado el tiempo que tardan los jóvenes en salir del desempleo. Asimismo, se evidencia que la probabilidad de conseguir un empleo depende de la zona de residencia y las mujeres jóvenes tienen menos probabilidades de emplearse. Adicionalmente, se encuentra que el deterioro de la salud mental de la población económicamente activa, a causa de la Covid-19, reduce la probabilidad de conseguir empleo.

El resto del artículo se estructura de la siguiente manera. En la primera sección se revisa la literatura relacionada con el desempleo juvenil, contextualizada por la situación de la mujer y las enfermedades mentales derivadas del desempleo en tiempos de pandemia. En la segunda se presentan los hechos estilizados sobre el desempleo en Cali. En la tercera se introduce la metodología de duración del desempleo. En la cuarta sección se discuten los resultados y en la última se presentan las conclusiones y las recomendaciones de política.

\section{REVISIÓN DE LITERATURA}

En el caso colombiano, son diversos los estudios que se han enfocado en analizar la participación de los jóvenes en el mercado laboral y las tasas de desempleo a las que estos se enfrentan. Entre ellos se encuentra el realizado por De La Hoz et al. (2012), quienes examinan las causas del desempleo juvenil, encontrando que dicho fenómeno se debe a la dinámica demográfica del país; a las brechas existentes entre oferta y demanda de trabajo; a la existencia del salario mínimo; y a la dinámica de la actividad económica. Por otro lado, los autores indican que la educación, como base de la teoría del capital humano, juega un papel importante en la inserción laboral y en la probabilidad de obtener trabajo. Finalmente, los autores proponen que el desempleo juvenil tiene consecuencias a largo plazo, impactando tanto la probabilidad de emplearse en el futuro como los ingresos a percibir.

Entre los trabajos más recientes se encuentra el realizado por Serna et al. (2019), el cual estudia la inserción al mercado de trabajo de los jóvenes en Colombia, individuos entre 14 y 28 años, usando datos de la Gran Encuesta Integrada de Hogares (GEIH) para el periodo 20082015. Los resultados muestran que la tasa global de participación aumentó, del 56,9\% en 2008 al 62,9\% en 2015, mientras que la tasa de desempleo disminuyó en el periodo, para situarse en el 16,3\% en 2015. Además, los autores encuentran que un alto nivel de educación 
no refleja un bajo nivel de desempleo, considerando que la tasa de desempleo afrontada por los jóvenes universitarios es superior a la que presentan los individuos sin ningún nivel educativo; específicamente, en 2015 la tasa de desempleo de los jóvenes universitarios fue del $15,9 \%$, mientras que la de los jóvenes sin ningún nivel educativo fue del $13,6 \%$. Finalmente, respecto al género, los resultados muestran una diferencia prolongada durante todo el periodo de estudio, donde la tasa de desempleo de las mujeres jóvenes es superior a la de los hombres, y la tasa global de participación inferior.

En cuanto al análisis de la duración del desempleo en Colombia, es posible encontrar estudios con diversos enfoques. Ejemplo de ello, es el estudio realizado por Viáfara y Uribe (2009), quienes examinan la duración del desempleo en Colombia y su relación con los canales de búsqueda, utilizando datos de la GEIH para el segundo trimestre de 2006. A través de la estimación de un modelo Weibull, los autores encuentran que los canales de búsqueda inciden en la duración del desempleo, específicamente, los resultados indican que el uso de las redes sociales como canal de búsqueda es ineficiente, mientras que el uso de canales formales, como las convocatorias y los avisos clasificados, facilitan la consecución de empleo. Respecto al género, se evidencia que la duración del desempleo de las mujeres es un 39\% superior que aquella de los hombres. Finalmente, en cuanto al capital humano, los resultados establecen que un año adicional de educación reduce la duración del desempleo en cerca de un $1 \%$.

En esta misma línea se encuentra el trabajo realizado por Jiménez (2012), quien analiza la eficiencia de los canales de búsqueda en términos de la duración del desempleo a través del uso de datos de la GEIH para el área metropolitana de Cali, Colombia, para los segundos trimestres de 2009 y 2010. A través de un análisis paramétrico y no paramétrico, se observa que ser hombre y poseer un mayor número de años de educación reduce la duración del desempleo. En cuanto a los canales de búsqueda, los resultados muestran que el uso de canales informales reduce la duración del desempleo, presentando un mayor índice de efectividad que los canales formales.

Por otro lado, Marcillo (2015) estudia la relación existente entre la duración del desempleo y el trabajo no remunerado para Colombia, utilizando datos de la GEIH de 2013. Los resultados indican que el trabajo no remunerado incide en la duración del desempleo, donde las mujeres, al dedicar un mayor tiempo al desarrollo de trabajo no remunerado y, por ende, disponer de menos tiempo para la realización 
de actividades de búsqueda de empleo, presentan una mayor probabilidad que los hombres de permanecer desempleadas.

Mora et al. (2017) analizan el desempleo juvenil y su duración en Cali, con datos por comuna de la Encuesta de Empleo y Calidad de Vida 2012-2013. Los resultados muestran que la probabilidad de salir del desempleo es menor cuanto mayor es el tiempo de búsqueda. Adicionalmente, se evidencia que los afrodescendientes e inmigrantes tienen una mayor probabilidad de seguir desempleados, al igual que las mujeres frente a los hombres. Por otro lado, los autores encuentran que el $25,8 \%$ de los jóvenes se pueden clasificar como "ninis", donde las mujeres presentan una probabilidad mayor de ser "ninis" que su contraparte los hombres.

Finalmente, Mora (2021b) analiza la participación laboral de los jóvenes en Buenaventura. Utilizando la geolocalización de 1094 jóvenes, estima un modelo espacial para las decisiones individuales de participar en el mercado laboral de Buenaventura. Sus resultados muestran que la decisión de participar en el mercado laboral se ve afectada por la decisión de participar de los jóvenes que se encuentran en su vecindario. De esta forma, existen efectos sociales en la participación laboral juvenil y las políticas públicas orientadas a los jóvenes deben partir de cómo los jóvenes interactúan entre ellos y cómo el comportamiento social afecta las decisiones de participar en el mercado laboral.

Sin embargo, la situación de los jóvenes en Cali no es la misma teniendo en cuenta el sexo. Las mujeres jóvenes tienen menos oportunidades que los hombres jóvenes y esta asimetría se ha intensificado por la crisis de la Covid-19 (Mora,2021 a) Situación, que parece ser un fenómeno generalizado a nivel mundial. En este sentido, Alon et al. (2020) analizan cómo la actual recesión económica causada por la Covid-19 afectó la desigualdad de género en el mercado laboral estadounidense, encontrando que las medidas de distanciamiento social tuvieron un gran impacto negativo en el sector de servicios, el cual concentra una alta proporción de empleo femenino.

Asimismo, Adams-Prassl et al. (2020) examinan el impacto de la pandemia de covid-19 en el mercado laboral de Estados Unidos, Reino Unido y Alemania, dejando ver que los trabajadores menos educados y las mujeres son los más afectados por la crisis en relación con la perdida de empleos, mientras que los jóvenes presentan una mayor probabilidad de experimentar una caída en sus ingresos.

Por su parte, el cierre de escuelas y guarderías aumentó las necesidades de cuidado infantil y del hogar, afectando principalmente a 
las madres trabajadoras, lo cual se evidencia en los aportes de Molina y Gonzales (2021), Farré et al. (2020), Alon et al. (2020) y OECD (2020), para los casos de Perú y Ecuador, España, Estados Unidos y la región $\mathrm{MENA}^{2}$, respectivamente. Finalmente, Ramos y Bolívar (2020) analizan la brecha de género en el mercado laboral colombiano durante la actual crisis derivada de la Covid-19, indicando que la pandemia ha contribuido al incremento de esta brecha.

Desde una perspectiva psicológica, el coste social de esta realidad es probable que sea sustancial. Investigaciones han demostrado que, más allá de la pérdida utilitarista del trabajo, el propio proceso de búsqueda de empleo puede provocar una disminución del bienestar psicológico (McKee-Ryan et al., 2005, Crayne, 2020), lo que agrava un conjunto de situaciones ya difíciles en tiempos de pandemia. Así, los efectos económicos derivados la pandemia, como el aumento de los despidos o la imposibilidad de trabajar con remuneración, la carga de los préstamos bancarios y el impago de los alquileres, suponen un aumento considerable de los niveles de estrés para la población, especialmente para los jóvenes (Extremera, 2020, Majeed, 2021).

Bajo este enfoque, Ghimire y Khanal (2021) sostienen que el estrés (causado por cambio en el patrón de vida y la expectativa no gestionada) y la ansiedad (desarrollada por el pesimismo, las pérdidas y la soledad) se relacionan con la persistencia y el aumento del desempleo de los jóvenes rurales en Nepal. Resultados similares a señalados anteriormente por Hagquist y Starrin (1996), quienes evidencian una relación explícita entre el desempleo y las enfermedades mentales de los jóvenes residentes de un pequeño municipio de Suecia. Por su parte, Khun et al. (2021) analiza las consecuencias de la crisis de la Covid-19 sobre el estrés y el bienestar en Suiza, encontrando que para los grupos más vulnerables: jóvenes, mujeres y desempleados, se reportó una disminución en la satisfacción con la vida, mientras que los niveles de estrés disminuyeron con mayor fuerza en las personas con mayores ingresos. Finalmente, Raynor y Panza (2020) describen los efectos de la Covid-19 sobre las personas que comparten una vivienda, que en su mayoría son jóvenes, observando aumentos en los niveles de estrés por vivienda y desempleo en los encuestados.

${ }^{2}$ El término MENA es un acrónimo del inglés "Middle East and North Africa”, que se refiera a la región del Medio oriente y norte de África. Esta región está compuesta por Argelia, Bahrein, Yibuti, Egipto, Irak, Jordania, Kuwait, Líbano, Libia, Mauritania, Marruecos, Omán, Autoridad Palestina, Qatar, Arabia Saudita, Siria, Túnez, Emiratos Árabes Unidos y Yemen. 


\section{EL DESEMPLEO DE LOS JÓVENES}

Las consecuencias económicas del coronavirus han llevado el mundo a terrenos desconocidos. Las medidas para controlar la propagación del virus (distanciamiento social y cuarentena principalmente) a partir del mes de febrero de 2020 han provocado el cierre o suspensión de las actividades económicas (con excepción de las declaradas como esenciales) y la pérdida de puestos de trabajo. Independientemente, uno de los grupos poblacionales que probablemente se ha visto más afectado por la crisis económica son los jóvenes.

La gráfica 1 recoge los principales efectos de la pandemia sobre el nivel de desempleo en tres países de América Latina (LATAM) y en las siete principales potencias económicas (G-7) durante 2020. Se destaca el significativo aumento del desempleo con respecto a los niveles de 2019, en especial de los jóvenes (de 15 a 24 años), cuyas tasas de desocupación media se incrementaron en 4,6 y 3,2 p.p. en los países de LATAM y del G-7 respectivamente; un aumento inferior a los 6,1 p.p. de los desocupados de mayor edad en LATAM, pero más del doble del registrado para los trabajadores de 25 a 74 años en el G-7, de 1,4 p.p.

\section{Gráfica 1}

Tasa de desempleo por grupos de edades, 2019-2020

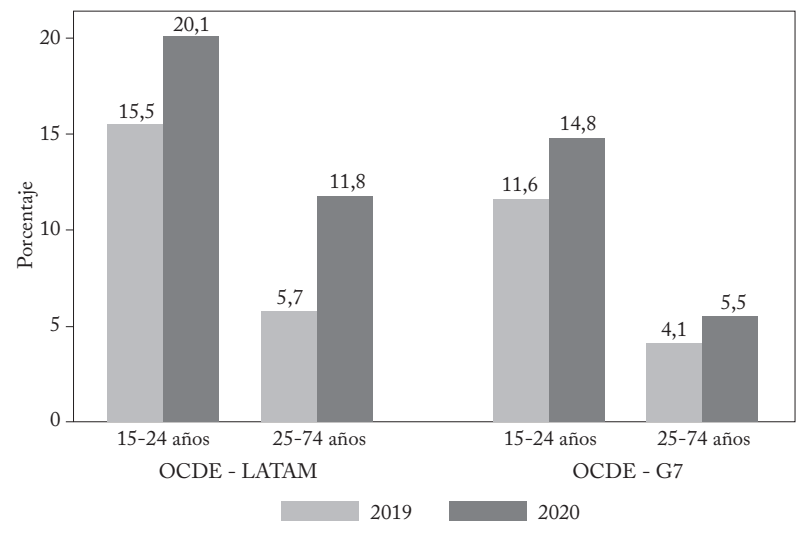

Las tasas corresponden a promedios simples. LATAM: Chile, Colombia y México. G-7: Estados Unidos, Japón, Alemania, Reino Unido, Francia, Italia y Canadá.

Fuente: OCDE (2021), elaboración propia.

En América Latina, el desempleo juvenil es muy diverso. Mientras que en Guatemala y México es relativamente bajo (inferior al 7\%), en Colombia, Argentina, Uruguay y Brasil fluctúa entre un 20\% y un 30\% 
(OIT, 2019). En el caso de los países latinoamericanos considerados, Colombia tuvo el mayor incremento (6,7 p.p.) en la desocupación juvenil durante el primer año de pandemia, del 20,8\% en 2019 al $27,5 \%$ en 2020, superior en 0,4 p.p. al de Chile en el mismo periodo (ver el cuadro 1 ).

Vale la pena señalar que la tasa de desempleo de los jóvenes en América Latina y el Caribe había aumentado más de 4 p.p. entre 2013 y 2016 (del 14\% al 18,3\%) y se estabilizó entre 2017 y 2019 (en el 19,5\%), mientras que el desempleo de los demás trabajadores pasó del 4,7\% en 2013 al 6,4\% en 2019, aumentado en promedio 1,5 p.p. como resultado de la desaceleración del crecimiento económico en la región (OIT, 2019). En tales situaciones, el peso de los ajustes suele afectar en mayor medida el empleo juvenil (Cepal, 2012; Henehan, 2020).

Cuadro 1

Tasa de desempleo por grupos de edades en tres países latinoamericanos 2019-2020. (Porcentajes)

\begin{tabular}{lcccccc}
\hline País & \multicolumn{2}{c}{2019} & \multicolumn{2}{c}{2020} & \multicolumn{2}{c}{ Diferencia (p.p.) } \\
\cline { 2 - 7 } & $15-24$ & $25-74$ & $15-24$ & $25-74$ & $15-24$ & $25-74$ \\
& años & años & años & años & años & años \\
\hline Chile & 18,6 & 6,1 & 24,8 & 9,6 & 6,3 & 3,5 \\
Colombia & 20,8 & 8,4 & 27,5 & 13,9 & 6,7 & 5,5 \\
México & 7,2 & 2,7 & 8,0 & N.D. & 0,8 & - \\
\hline
\end{tabular}

Fuente: OCDE (2021), elaboración propia.

Sin embargo, el aumento de la desocupación entre los jóvenes observado durante la pandemia, al igual que el de los adultos, ha sido inferior al esperado debido al significativo deterioro de la actividad económica, registrado especialmente en el segundo trimestre de 2020 , permitiendo la transición del empleo a la inactividad como consecuencia directa de las medidas de confinamiento en los hogares y de la paralización de las actividades económicas.

En Colombia, la pérdida de empleo de los jóvenes se refleja en una reducción de 12,5 p.p. en la tasa de ocupación y en una disminución de 8 p.p. en la tasa global de participación durante el segundo trimestre de 2020 respecto del mismo periodo de 2019, atenuando el impacto de la pérdida de empleos en la tasa de desocupación.

\section{LA SITUACIÓN DE LOS JÓVENES DE CALI ANTES Y DURANTE LA PANDEMIA}

La situación del mercado laboral en Cali muestra patrones similares a los exhibidos por las demás economías. El cuadro 2 presenta los principales indicadores del mercado laboral de los jóvenes. 
Las cifras anteriores dejan ver que en febrero de 2021 los jóvenes presentaron una tasa de desempleo casi 9 p.p. superior a la tasa de desempleo de la ciudad (20,7\%), y una tasa de ocupación 8 p.p.inferior que la reportada para Cali $(53,4 \%)$.

Además, entre febrero de 2020 y febrero de 2021 se deterioraron las tasas de ocupación de hombres y mujeres, 8 p.p. y 6,4 p.p. respectivamente, con un incremento de sus tasas de desempleo superior a 11 p.p. En cuanto a las brechas de género en el nivel de desocupación, se evidencia que, para febrero de 2021, las mujeres presentaron una tasa superior a los hombres en aproximadamente 13,9 p.p. Por otro lado, los jóvenes afrodescendientes han sido fuertemente golpeados por la pandemia, como indican el aumento de su tasa de desempleo y la fuerte caída de su tasa de ocupación, las cuales superan los 14 p.p.

Cuadro 2

Indicadores mercado laboral juvenil, Cali y Área Metropolitana (Cali AM) (Porcentajes)

\begin{tabular}{lcccccc}
\hline \multirow{2}{*}{ Cali AM } & \multicolumn{2}{c}{ Febrero de 2020} & \multicolumn{2}{c}{ Febrero de 2021 } & \multicolumn{2}{c}{ Diferencia (p.p.) } \\
\cline { 2 - 7 } & TO & TD & TO & TD & TO & TD \\
\hline Total & 53,2 & 18,1 & 45,2 & 29,6 & $-8,0$ & 11,5 \\
Hombres & 57,8 & 10,5 & 51,3 & 23,0 & $-6,4$ & 12,6 \\
Mujeres & 48,7 & 25,4 & 39,0 & 36,9 & $-9,7$ & 11,4 \\
Afrodescendientes & 56,7 & 20,6 & 42,1 & 35,4 & $-14,5$ & 14,8 \\
\hline
\end{tabular}

Fuente: Dane-GEIH, 2020-2021, elaboración propia.

Ahora bien, con relación a la distribución espacial del desempleo juvenil en la ciudad de Cali en tiempos de pandemia, la información reportada en el cuadro 3 permite evidenciar que, para el total de jóvenes, la zona Urbana Oriente de la ciudad presentó la mayor tasa de desempleo, $43 \%$. Esta zona se encuentra compuesta por las comunas 6, $7,13,14,15,16$ y 21 , dentro de las cuales destacan las comunas 13,14 , 15 y 21 , que conforman el sector del Distrito de Aguablanca, uno de los sectores más deprimidos y con mayores problemáticas socioeconómicas y de violencia de la ciudad. La mayoría de los barrios del Distrito de Aguablanca se caracterizan por su bajo nivel socioeconómico, a la par de un escaso nivel educativo, donde aproximadamente el 65\% de sus residentes no superan el nivel de educación media, dificultando su inserción a las dinámicas laborales de la ciudad.

La zona Centro Ampliado y Peri-centro, compuesta por las comunas 3, 4, 8, 9, 10, 11 y 12, presentó la segunda mayor desocupación juvenil en la ciudad, el 35,1\%, seguida de las zonas Corredor Norte-Sur (comunas 2, 5, 17, 19 y 22) y Ladera (comunas 1, 18 y 
20), con tasas del 25,2\% y el 21,7\% respectivamente. Ahora bien, el Corredor Norte-Sur, conformado mayoritariamente por barrios de alto nivel económico y social, reflejado en uno de cada cuatro jóvenes residentes con título universitario, presentó una tasa de desempleo 3,5 p.p. inferior a la exhibida por la zona de Ladera, cuyos barrios más representativos: Siloé, Belén y Terrón Colorado, se caracterizan por albergar condiciones de pobreza similares a las del Distrito de Aguablanca.

Cuadro 3

Tasas de desempleo de los jóvenes en Cali (Porcentajes)

\begin{tabular}{lccccccc}
\hline Zona & Total & Mujeres & $\begin{array}{c}\text { Mujer } \\
\text { afro }\end{array}$ & $\begin{array}{c}\text { Mujer } \\
\text { indígena }\end{array}$ & Hombres & $\begin{array}{c}\text { Hombre } \\
\text { afro }\end{array}$ & $\begin{array}{c}\text { Hombre } \\
\text { indígena }\end{array}$ \\
\hline $\begin{array}{l}\text { Ladera } \\
\text { Urbana Corredor }\end{array}$ & 21,7 & 25,4 & N.D. & 38,9 & 18,3 & 29,8 & N.D. \\
$\begin{array}{l}\text { Norte-Sur } \\
\text { Centro Ampliado }\end{array}$ & 28,7 & 21,6 & 55,3 & 21,8 & 17,3 & 29,0 \\
$\begin{array}{l}\text { y Peri-centro } \\
\text { Urbana Oriente }\end{array}$ & 43,1 & 37,2 & 46,1 & 16,7 & 33,4 & 36,4 & 8,6 \\
Total & 54,1 & 63,6 & 45,5 & 33,3 & 40,4 & 45,1 \\
\hline
\end{tabular}

Fuente: Proyecto Fortalecimiento del Sistema de C\&CTI del Valle del Cauca: hacia una Economía del Conocimiento y Encuesta Multipropósito de Empleo y Calidad de Vida, agosto-noviembre de 2020, elaboración propia.

Un aspecto que cabe destacar es la notoria segmentación de género existente en el mercado laboral juvenil, donde las brechas de desocupación fluctuaron entre 4 p.p. y 20 p.p. en favor de los hombres según la zona de residencia. Para el total de la ciudad, las mujeres alcanzaron una tasa de desempleo 12 p.p. mayor que la de los hombres.

Al considerar las diferencias étnicas, la menor brecha de desempleo entre mujeres y hombres afrodescendientes se presentó en la zona Corredor Norte-Sur, donde la diferencia se situó alrededor de 4 p.p. en favor de los segundos. De manera análoga, esta zona presentó la mayor brecha (26,3 p.p.) entre mujeres y hombres indígenas.

Conforme a lo esperado, la zona Urbana Oriente concentró los mayores niveles de desempleo según etnia y género, con excepción de mujeres indígenas, lo cual influye significativamente en el comportamiento del mercado laboral de la ciudad.

\section{METODOLOGÍA}

E1 análisis de la duración del desempleo se realiza de dos formas. En primer lugar, se realiza un análisis no paramétrico basado en el 
método de Kaplan y Meier (1958), el cual permitirá establecer cuán rápido los jóvenes caleños pueden salir del desempleo.

En segundo lugar, se efectúa un análisis paramétrico en el cual se consideran una serie de características socioeconómicas de los individuos que integran el mercado laboral de Cali y que podrían influir en la duración del desempleo. En este contexto, se sigue la notación y línea argumental de Mora et al. (2017), cuyo desarrollo estadístico se presenta a continuación:

Sea una muestra de jóvenes que cae al desempleo en un mes, la tasa de riesgo mensual discreta del joven en ese mes, y un vector de covariantes entre los cuales están los periodos que lleva en desempleo o el tipo de estudio (diploma que ha obtenido). La tasa de riesgo mensual discreta vendrá dada por:

$h_{j}\left(X_{i j}\right)=1-\exp \left\{-\exp \left[X_{i j}^{\prime} \beta+\gamma_{j}+\log \left(\varepsilon_{i}\right)\right]\right\}$

$\gamma_{j}=\log \int_{a_{j-1}}^{a_{j}} \lambda_{0}(\tau) d \tau$

Donde $\varepsilon_{i}$ sigue una distribución gamma con media unitaria y varianza $\sigma^{2} \equiv \mathrm{v}, \mathrm{y} \gamma_{j}$ es el logaritmo de la integral del riesgo básico sobre el intervalo relevante $a_{j}$. La función de verosimilitud en logaritmos tiene la siguiente expresión:

$\log L=\sum_{i=1}^{\mathrm{N}} \log \left\{\left(1-d_{i}\right)^{*} \mid A_{i}+d_{i}^{*} \beta_{i}\right\}$

Donde:

$A_{i}=\left[1+v \sum_{j=1}^{t_{i}} \exp \left[X_{i j}^{\prime} \beta+\theta(j)\right]\right]^{-(1 / v)}$

Por su parte,

$\beta_{i}=\left[1+\mathrm{v} \sum_{j=1}^{t_{i}-1} \exp \left[X_{i j} \beta+\theta(j)\right]\right]^{-(1 / v)}-A_{i} \operatorname{Si} t_{i}>1$

Si $t_{i}=1, \beta_{i}=1-A_{i}$

$N$ es el tamaño de la muestra, $t_{i}$ es el número de meses que el joven $i$ se encuentra en riesgo, $d_{i}$ es una variable dicotómica que toma el valor de 1 para el mes en el que se produce un cambio de posición (sale del desempleo) y cero en los meses restantes, y $\theta(j)$ es una función que describe la dependencia de la duración en la tasa de riesgo incluyendo la especificación no paramétrica del riesgo básico (Jenkins, 1995). 


\section{RESULTADOS}

Para analizar los efectos de la pandemia en la duración del desempleo de los jóvenes caleños, se los datos de la $\mathrm{GEIH}^{3}$ de febrero 2020 y 2021, y los de la Encuesta Multipropósito de Empleo y Calidad de Vida, realizada entre agosto y noviembre de 2020 por el Componente de Medición Económica del proyecto Fortalecimiento del Sistema de C\&CTI del Valle del Cauca: hacia una Economía del Conocimiento ${ }^{4}$.

Gráfica 2

Tasa de salida del desempleo juvenil para Cali-AM

K-M, febrero de 2020

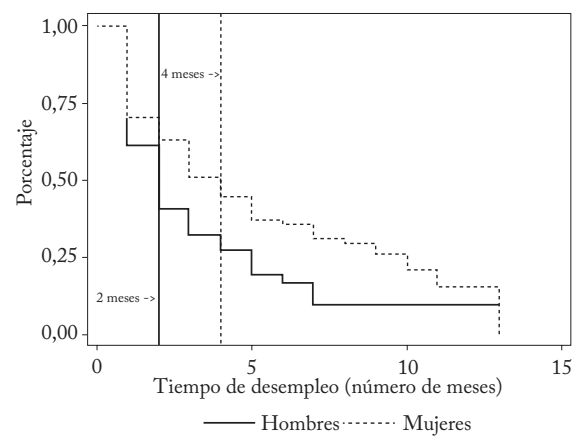

(B) K-M, febrero de 2021

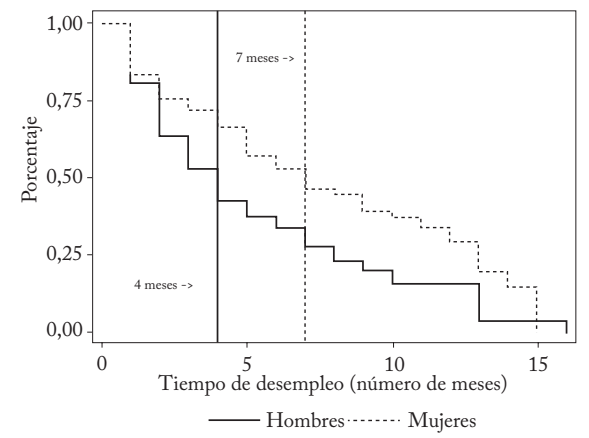

Fuente: Dane-GEIH, 2020-2021, elaboración propia.

La gráfica 2 presenta la duración del desempleo de los jóvenes en Cali-AM utilizando el método no paramétrico de Kaplan-Meier (K-M). Este método permite determinar la magnitud de la duración del desempleo.

Se puede observar que la tasa de salida del desempleo ha sido superior para las mujeres que para los hombres, es decir, las mujeres tardan más tiempo en conseguir empleo, situación que se ha agudizado en tiempos de pandemia. Específicamente, antes de la actual crisis sanitaria aproximadamente el 50\% de los hombres jóvenes conseguía

3 La GEIH es una encuesta multipropósito realizada por el Dane, que monitorea el mercado laboral y proporciona información mensual del país, los departamentos y las principales ciudades capitales.

${ }^{4}$ El trabajo de campo para la obtención de los datos fue realizado por el Centro Nacional de Consultoría (CNC). El marco de muestreo utilizado es el Marco Geoestadístico Nacional provisto por el Dane y el margen de error es de $1,4 \%$ con un nivel de confianza del 95\%. E1 Proyecto mencionado es financiado con recursos del Sistema General de Regalías, formulado por la Red de Universidades para la innovación del Valle del Cauca (RUPIV) y apoyado por la Gobernación. Para más información del proyecto, ver [https:// valledelconocimiento.com]. 
empleo en los 2 primeros meses y las mujeres en los primeros 4 . Durante la pandemia, esta misma proporción de hombres conseguía empleo en los 4 primeros meses y las mujeres en los primeros 7. En otras palabras, como efecto de la pandemia, se duplicó el tiempo en el cual los jóvenes salen del desempleo.

Ahora bien, la gráfica K-M solo establece una relación no paramétrica que permite definir la proporción de jóvenes que pasan del desempleo al empleo en un momento del tiempo determinado. Para indagar las condiciones en que los jóvenes salen del desempleo a un empleo se consideró el modelo de la ecuación (2). Como covariantes se incluyeron las siguientes variables: (a) 1-3 Meses es una variable dicotómica que toma el valor de 1 si el individuo duró desempleado entre uno y tres meses; (b) 4-6 Meses es una variable binaria que toma el valor de 1 si el individuo duró desempleado entre cuatro y seis meses; (c) Esdeprcovid-19 es una variable dicotómica que toma el valor de 1 si la persona tuvo estrés o depresión como resultado de la Covid-19; (d) Diploma Tec/Tecnolg es una variable dicotómica que toma el valor de 1 cuando la persona tiene un diploma técnico o tecnológico; (e) Diploma Universitario es una variable binaria que toma valor de 1 cuando la persona tiene un diploma universitario; y (f) Mujer es una variable dicotómica que toma el valor de 1 cuando el individuo es mujer.

El cuadro 4 presenta las estimaciones. Los resultados indican que a medida que pasa el tiempo, 3 y 6 meses, se reducen las posibilidades de los jóvenes para conseguir empleo. Los jóvenes tienen una menor probabilidad de emplearse en los tres primeros meses que los no jóvenes $(0,19$ vs. 0,56$)$, es decir, estos últimos tienen una probabilidad 2,9 veces mayor. El nivel de formación educativa tiene una influencia significativa en la probabilidad de conseguir empleo. Los jóvenes con título técnico o tecnológico tienen una probabilidad casi 2 veces mayor que los que no poseen dicho título, mientras que los jóvenes universitarios tienen 2,5 veces más probabilidades de encontrar un empleo. Este patrón es análogo al del total de la población caleña, para la cual es mayor la probabilidad de emplearse cuanto mayor es su formación educativa.

Asimismo, se observan patrones de segregación de género. Las jóvenes tienen 0,41 veces menos probabilidades de emplearse que los jóvenes, un valor semejante a la brecha existente en la ciudad. Cuando se consideran las personas que han sufrido de estrés o depresión a causa de la Covid-19, se encuentra que las no jóvenes tienen 0,78 veces menos probabilidades de encontrar empleo; para las jóvenes no 
hubo un efecto estadísticamente significativo. Para complementar esos hallazgos se presentan los resultados del análisis espacial considerando las diversas zonas de la ciudad de Cali. La gráfica 3 muestra la duración del desempleo de los jóvenes según el enfoque K-M.

Cuadro 4

Modelo paramétrico de duración Cali- $\mathrm{AM}^{5}$

\begin{tabular}{lccc}
\hline Variables & Todos & No jóvenes & Jóvenes \\
\hline 1-3 Meses & $0,4212^{* * * *}$ & $0,5635^{* * * *}$ & $0,1947^{* * * *}$ \\
& $(0,0110)$ & $(0,0165)$ & $(0,0113)$ \\
4-6 Meses & $0,3951^{* * * *}$ & $0,4539^{* * *}$ & $0,2853^{* * *}$ \\
& $(0,0163)$ & $(0,0210)$ & $(0,0260)$ \\
Esdeprcovid-19 & $0,8572^{* * *}$ & $0,7800^{* * *}$ & 0,9534 \\
& $(0,0377)$ & $(0,0372)$ & $(0,1036)$ \\
Diploma Téc/Tecnolg & $1,2416^{* * *}$ & 1,0354 & $1,8969^{* * *}$ \\
& $(0,0624)$ & $(0,0566)$ & $(0,2296)$ \\
Diploma Universitario & $1,7115^{* * *}$ & $1,3443^{* * * * *}$ & $2,4891^{* * * *}$ \\
& $(0,0900)$ & $(0,0738)$ & $(0,3844)$ \\
Mujer & $0,4420^{* * *}$ & $0,4454^{* * *}$ & $0,4060^{* * * *}$ \\
& $(0,0156)$ & $(0,0174)$ & $(0,0338)$ \\
\hline Gamma & 1,3968 & 1,2387 & 1,8909 \\
S.E. Gamma & 0,037 & 0,037 & 0,12 \\
LR Test Gamma & 8952 & 7075 & 1683 \\
N & 58.044 & 44.896 & 13.148 \\
\hline
\end{tabular}

Fuente: Dane-GEIH (febrero 2021), elaboración propia
Nota: Errores estándar en paréntesis. Niveles de significancia: ${ }^{*}<0,1 ; * *<0,05 ;{ }^{* * *}<0,01$.

Tales resultados sugieren que en la zona Urbana Oriente solo habían salido del desempleo el 25\% de los jóvenes después de 5 meses. En general para las distintas zonas, el $50 \%$ de los jóvenes solo pudieron salir del desempleo después de 9 meses, exceptuando los jóvenes residentes en la zona de Ladera donde el 50\% de estos tardaron 6 meses y, al cabo de 13 meses, uno de cada cuatro de estos jóvenes se encontraba aún sin salir del desempleo. Por último, la salida del desempleo fue muy lenta entre los 9 y 13 meses, incluso encontrándose una proporción significativa de jóvenes desempleados después de los 20 meses, concretamente en las zonas Urbana Oriente (17\%) y Centro Ampliado y Peri-Centro (9\%).

${ }^{5}$ Los resultados se reportan conforme a la razón de Hazard, , por lo cual valores menores que 1 reducen la probabilidad y valores mayores que 1 aumentan la probabilidad. 
Gráfica 3

Tasa de salida del desempleo juvenil, Cali

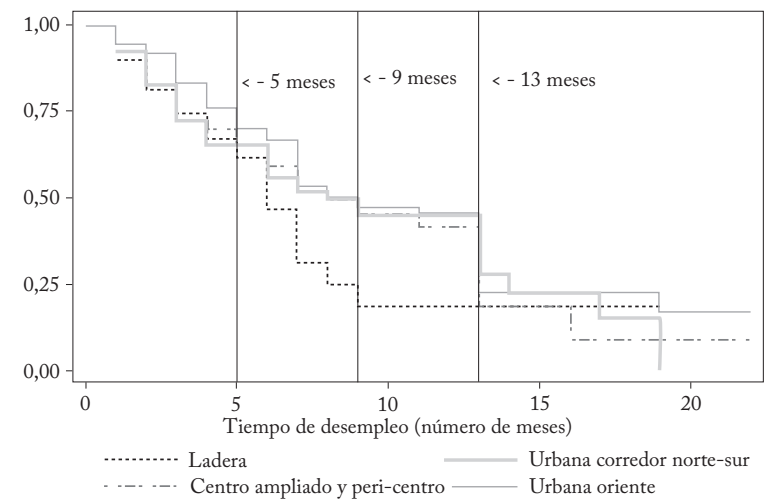

Fuente: Proyecto Fortalecimiento del Sistema de C\&CTI del Valle del Cauca: hacia una Economía del Conocimiento y Encuesta Multipropósito de Empleo y Calidad de Vida, agosto-noviembre de 2020; elaboración propia.

E1 cuadro 5 presenta los resultados del modelo paramétrico de duración del desempleo, para las zonas más vulnerables de la ciudad. En este caso se emplean las siguientes covariantes: (a) 1 Mes, 2 Meses y 3 Meses, son variables dicotómicas que toman el valor de 1 si el individuo duró desempleado un mes, dos meses y tres meses; (b) Zona Ladera y Zona Urbana Oriente, son variables binarias que toman el valor de 1 si el individuo reside en esas zonas; (c) Diploma Tec/Tecnolg y Diploma Universitario, son variables dicotómicas que toman el valor de 1 cuando la persona tiene diploma técnico/tecnológico y universitario; (d) Mujer, es una variable dicotómica que toma el valor de 1 si la persona es mujer; (e) Afrodescendiente, es una variable binaria que toma el valor de 1 cuando el individuo es afrodescendiente; y (f) Ecoadcontaf, es una variable dicotómica que toma el valor de 1 si el área formación del individuo es Economía, Administración, Contaduría y afines.

A medida que pasa el tiempo la probabilidad de conseguir empleo es mucho menor. En general, los individuos que llevan un mes en desempleo tienen 0,09 veces menos probabilidades de conseguir empleo, mientras que aquellos con 3 meses tienen 0,47 veces menos probabilidades de salir del desempleo.

A medida que pasa el tiempo la probabilidad de conseguir empleo es mucho menor. En general, los individuos que llevan un mes en desempleo tienen 0,09 veces menos probabilidades de conseguir empleo, mientras que aquellos con 3 meses tienen 0,47 veces menos probabilidades de salir del desempleo. 
En cuanto a las zonas, los residentes de Ladera tienen 0,4 veces menos probabilidades de salir del desempleo que aquellos que residen en otras zonas, mientras que para los jóvenes habitar en esta zona no tiene un efecto estadísticamente significativo. Por otro lado, los individuos que viven en la zona Urbana Oriente tienen 0,43 veces menos probabilidades de salir del desempleo y los jóvenes residentes en esta zona presentan 0,36 veces menos probabilidades de emplearse que aquellos que no viven en esas comunas.

Cuadro 5

Modelo paramétrico de duración, Cali

\begin{tabular}{lcccc}
\hline Variables & Todos & No jóvenes & Jóvenes & JovProf \\
\hline 1 mes & $0,0881^{* * * *}$ & $0,0954^{* * * *}$ & $0,0501^{* * * *}$ & $0,0118^{* * *}$ \\
& $(0,0064)$ & $(0,0075)$ & $(0,0100)$ & $(0,0067)$ \\
& $0,2858^{* * * *}$ & $0,3140^{* * * *}$ & $0,1853^{* * * *}$ & $0,0821^{* * *}$ \\
& $(0,0282)$ & $(0,0340)$ & $(0,0452)$ & $(0,0525)$ \\
meses & $0,4733^{* * * *}$ & $0,4775^{* * *}$ & $0,4514^{* * * *}$ & 0,4033 \\
& $(0,0460)$ & $(0,0525)$ & $(0,0955)$ & $(0,2089)$ \\
Zona Ladera & $0,4055^{* * * *}$ & $0,3725^{* * * *}$ & 0,6166 & \\
& $(0,0550)$ & $(0,0559)$ & $(0,2068)$ & \\
Zona Urbana Oriente & $0,4312^{* * * *}$ & $0,4522^{* * * *}$ & $0,3597^{* * * *}$ & \\
& $(0,0386)$ & $(0,0444)$ & $(0,0800)$ & \\
Diploma técnico/tecnológico & $1,5587^{* * * *}$ & $1,5984^{* *}$ & $1,9138^{*}$ & \\
& $(0,1962)$ & $(0,2353)$ & $(0,5156)$ & \\
Diploma universitario & $2,2716^{* * * *}$ & $2,2876^{* * * *}$ & $1,9393^{*}$ & $6,3198^{*}$ \\
& $(0,2888)$ & $(0,3129)$ & $(0,6925)$ & $(4,5503)$ \\
Mujer & $0,3686^{* * * *}$ & $0,3710^{* * * *}$ & $0,3377^{* * * *}$ & $0,3204^{*}$ \\
& $(0,0306)$ & $(0,0335)$ & $(0,0735)$ & $(0,1807)$ \\
Afrodescendiente & $0,7328^{* *}$ & $0,7812^{*}$ & 0,6124 & 1,1445 \\
& $(0,0806)$ & $(0,0940)$ & $(0,1698)$ & $(0,8906)$ \\
Ecoadcontaf & & & & $4,5622^{*}$ \\
& & & & $(3,2604)$ \\
\hline Gamma & 2,1509 & 2,0798 & 2,5768 & 3,6916 \\
S.E. gamma & 0,1231 & 0,1301 & 0,3597 & 1,0455 \\
LR Test gamma & 3566,47 & 3087,04 & 421,69 & 197,88 \\
N & 25.099 & 20.980 & 4.119 & 877 \\
\hline
\end{tabular}

Los errores estándar van entre paréntesis. Niveles de significancia: ${ }^{*}<0.1 ; * * 0.05 ;{ }^{* * * *}<0.01$.

Fuente: Proyecto "Fortalecimiento del Sistema de C\&CTI del Valle del Cauca: hacia una Economía del Conocimiento" - Encuesta Multipropósito de Empleo y Calidad de Vida, agosto-noviembre de 2020; elaboración propia.

Las personas que lograron titularse en educación superior tienen más posibilidades de salir del desempleo, presentando 1,56 y 2,27 veces más probabilidades para aquellos que cuentan con un título técnico/ 
tecnológico y universitario respectivamente. No obstante, los jóvenes universitarios tienen escasamente mayores probabilidades de salir del desempleo que quienes tienen títulos técnicos o tecnológicos.

Por su parte, las mujeres presentan, en general, 0,37 veces menos probabilidades que los hombres de salir del desempleo, situación que se atenúa levemente al tratarse de las jóvenes. Respecto a la etnia, se encuentra que los no jóvenes afrodescendientes tienen 0,78 veces menos posibilidades de emplearse respecto a las demás etnias, efecto que se pierde en la población joven.

Finalmente, los jóvenes formados en áreas del conocimiento como economía, administración, contaduría y afines pueden moverse mejor en el mercado laboral, lo que implica que tienen 4,56 veces más probabilidades de salir del desempleo.

\section{CONCLUSIONES}

Este artículo examina los efectos de la pandemia de Covid-19 en la duración del desempleo juvenil en la ciudad de Cali, analizando un conjunto de características socioeconómicas individuales que influyen en el tiempo que tardan los jóvenes en emplearse. Con ese fin se utiliza información oficial y privada sobre el mercado laboral juvenil antes y durante el primer año de la pandemia, que permite el análisis espacial por zonas urbanas y, así entender mejor este fenómeno para hacer recomendaciones de política que lo enfrenten de manera eficiente.

La desaceleración económica observada durante el 2020, como resultado de la pandemia, afectó en mayor medida a los jóvenes en Cali. Para febrero de 2021, la tasa de desocupación de los jóvenes se situó en 29,6\%, 9 p.p. superior a la tasa de la ciudad; mientras que la tasa de ocupación juvenil fue del 45,2\%, 8 p.p. inferior a la de Cali.

Los principales hallazgos de este estudio sugieren de manera altamente significativa que cuanto mayor es el tiempo de búsqueda de empleo menor es la probabilidad de salir del desempleo, encontrándose brechas en favor de los no jóvenes, con 2,9 veces más probabilidades de encontrar empleo durante los tres primeros meses que los jóvenes. Estos resultados vendrían dados por el menor nivel de capital humano que presenta la media de la fuerza laboral de los jóvenes, reafirmando la influencia que ejercen la educación y la experiencia laboral sobre el desempleo y la velocidad de su recuperación. Más aún, se encontró que los jóvenes con educación superior certificada tienen entre 1,9 y 2,5 veces más probabilidades de salir del desempleo en época de crisis. 
Por otra parte, durante la crisis se continuaron evidenciando las disparidades de género en el mercado laboral, donde las mujeres jóvenes exhiben tasas de desempleo más altas que las de los hombres y tasas de ocupación inferiores. Adicionalmente, en cuanto a la duración del desempleo, las mujeres jóvenes tardan en promedio el doble de tiempo en emplearse que su contraparte masculina, con 0,4 veces menos probabilidades de conseguir empleo.

Otro de los grupos poblacionales fuertemente afectados durante la crisis son los jóvenes afrodescendientes, quienes experimentaron un aumento en su tasa de desempleo al tiempo de una caída en su tasa de ocupación, ambas superando los 14 p.p.

Adicionalmente, se analizó la distribución del desempleo juvenil por zonas urbanas, con resultados interesantes. En particular, la zona de Ladera, la cual presenta condiciones similares a las registradas en el Distrito de Aguablanca, concentró el menor nivel de desocupación en la ciudad. Por otro lado, en las zonas de mayor y menor nivel socioeconómico se encontraron resultados conforme a lo esperado. Así, la zona Corredor Norte-Sur exhibió una tasa de desempleo inferior en 9,5 p.p. a la registrada en el total de la ciudad, mientras que esta tasa fue superior en 8,3 p.p. en la zona Urbana Oriente (caracterizada por concentrar la mayor proporción de personas en condición de pobreza y vulnerabilidad en la ciudad). En términos de la duración del desempleo, los jóvenes residentes en la zona Urbana Oriente tienen 0,36 veces menos probabilidades de emplearse que aquellos residentes en las demás zonas de la ciudad.

El impacto sobre el empleo y la desocupación juvenil en el actual contexto económico recesivo amerita respuestas urgentes en materia de política pública. Los hallazgos anteriores ratifican la importancia de la educación en los jóvenes como medio eficaz para salir del desempleo, resultados en línea con los expuestos por Mora et al. (2017) y Mora (2021). Sin embargo, las políticas públicas para los jóvenes deben estar orientadas, en primer lugar, a mejorar la inserción de las mujeres jóvenes en el mercado laboral. En segundo lugar, al reconocimiento de las particularidades del territorio (p. ej., las necesidades específicas de capital humano y la posibilidad de adaptación entre ocupaciones a las necesidades de las empresas a través de la interdisciplinariedad). En tercer lugar, a la identificación de los problemas espaciales que existen en la ciudad de Cali, haciendo hincapié en la heterogeneidad de sus comunas, lo cual permitiría una mayor inserción de los jóvenes al mercado laboral de Cali derivado del enfoque diferencial territorial. En cuarto lugar, al reconocimiento de una 
política pública de salud mental para los jóvenes ya que debido a la pandemia el estrés y la depresión han reducido la probabilidad de salir del desempleo. Finalmente, en la ciudad de Cali las políticas públicas se deben focalizar en contrarrestar el de desempleo de los jóvenes en sus dos primeros meses, dado que la probabilidad de conseguir trabajo se reduce a medida que pasa el tiempo.

\section{REFERENCIAS BIBLIOGRÁFICAS}

Adams-Prassl, A., Boneva, T., Golin, M., y Rauh, C. (2020). Inequality in the impact of the coronavirus shock: Evidence from real time surveys. IZA Institute of labor Economics. Discussion Paper, 13183.

Alon, T., Doepke, M., Olmstead-Rumsey, J. y Tertilt, M. (2020). The impact of COVID-19 on gender equality. NBER Working Paper 26947.

Arango, L. E. y Ríos, A. M. (2015). Duración del desempleo en Colombia: género, intensidad de búsqueda y anuncios de vacantes. Borradores de Economía, 866, 1-44.

Castillo, C. D. y García, J. (2019). Desempleo juvenil en Colombia ¿la educación importa? Revista Finanzas y Política Económica, 11(1), 101127. http://doi.org/10.14718/revfinanzpolitecon.2019.11.1.7

Cepal. (2012). El desempeño del empleo juvenil en la crisis y en la etapa de recuperación, Coyuntura Laboral en América Latina y el Caribe, No. 7, Santiago de Chile.

Crayne, M. P. (2020). The traumatic impact of job loss and job search in the aftermath of COVID-19. Psychological Trauma: Theory, Research, Practice, and Policy, 12(S1), S180-S182. https://doi.org/10.1037/ tra0000852

De la Hoz, F. J., Quejada, R., y Yánez, M. (2012). E1 desempleo juvenil: problema de efectos perpetuos. Revista Latinoamericana de Ciencias Sociales, Niñez y Juventud, 10(1), 427-439.

Extremera, N. (2020). Coping with the stress caused by the COVID-19 pandemic: future research agenda based on emotional intelligence. International Journal of Social Psychology, 35(3), 631-638. https://doi. org/10.1080/02134748.2020.1783857

Farré, L., Fawaz, Y., González, L., y Graves. J. (2020). How the COVID-19 Lockdown Affected Gender Inequality in Paid and Unpaid Work in Spain. IZA Institute of Labor Economics Discussion Paper, 13434.

Ghimire S. y Khanal J. (2021). COVID-19 and youth: Uncertainty, stress, anxiety. Youth Voice Journal, 11, 1-27.

Hagquist, C. y Starrin, B. (1996). Youth unemployment and mental healthgender differences and economic stress. Scandinavian Journal of Social Welfare, 5(4), 215-228. https://doi.org/10.1111/j.1468-2397.1996. tb00148.x

Henehan, K. (2020). Class of 2020: Education leavers in the current crisis, mayo, [https://www.resolutionfoundation.org/app/uploads/2020/05/ Class-of-2020.pdf].

Jenkins, S.P. (1995). Easy estimation methods for discrete-time duration models. Oxford Bulletin of Economics and Statistics, 57(1), 129-138 
Jiménez, D. M. (2012). Búsqueda de empleo y duración del desempleo en el área metropolitana de Cali: un recuento para los segundos trimestres de 2009 y 2010. Sociedad y economía, (22),163-186.

Kaplan, E. L. y Meier, P. (1958). Nonparametric estimation from incomplete observations. Journal of the American Statistical Association, 53, 457-481.

Kuhn, U., Klaas, H. S., Antal, E., Dasoki, N., Lebert, F., Lipps, O., Monsch, G. A., Refle, J. E., Ryser, V. A., Tillmann, R. y Voorpostel, M. (2021). Who is most affected by the Corona crisis? An analysis of changes in stress and well-being in Switzerland. European Societies, 23(sup1), S942-S956. https://doi.org/10.1080/14616696.2020.1839671

Majeed, B. I. (2021). Youth and COVID-19: Situational analysis. Indian Journal of Social Work, 82(2):235-246.

Marcillo, E.V. (2015). El trabajo no remunerado como determinante de la duración del desempleo en Colombia: un análisis a nivel de género. Archivos de Economía, 423.

McKee-Ryan, F., Song, Z., Wanberg, C. R., y Kinicki, A. J. (2005). Psychological and physical well-being during unemployment: a metaanalytic study. Journal of applied psychology, 90(1), 53-76. https://doi. org/10.1037/0021-9010.90.1.53

Molina, R. E., y Gonzales, J. M. (2021). Incremento de la vulnerabilidad de los derechos de la mujer durante la pandemia: escenario ecuatoriano y peruano. Revista Jurídica Científica SSIAS, 14(1). https:// doi.org/10.26495/rcs.v14i1.1652

Mora, J. J. (2021a). Análisis del desempleo y la ocupación después de una política estricta de confinamiento por COVID-19 en Cali. Lecturas de Economia, 94, 165-193, [https://doi.org/10.17533/udea.le.n94a342002].

Mora, J. J. (2021b). Spatial social effects on the decision to participate in the youth labor market in a developing country. Journal of Regional Research, 51(3), 61-80.

Mora, J. J., Caicedo, C. y González, C. G. (2017). Duración del desempleo de los jóvenes y los "ninis" en Cali, Colombia. Revista de Economía Institucional, 19(37), 167-184.

OECD. (2020). COVID-19 crisis in the MENA region: Impact on gender equality and policy responses, [https://read.oecd-ilibrary.org/ view/?ref=134_134470-w95kmv8khl\&title=COVID-19-crisis-inthe-MENA-region-impact-on-gender-equality-and-policy-responses].

OIT. (2019). Informe laboral: Panorama laboral juvenil en América Latina y el Caribe, panorama laboral 2019: América Latina y el Caribe, Lima, enero.

Ramos, C. I., y Bolívar, M. C. (2020). Brecha de género en el mercado laboral colombiano en tiempos de la Covid-19. Semestre Económico, 23(55), 285-312, [https://doi.org/10.22395/seec.v23n55a13].

Raynor, K., \& Panza, L. (2020). The impact of COVID-19 on Victorian Share Households, [https://minerva-access.unimelb.edu.au/bitstream/ handle/11343/242428/The-impact-of-COVID-19-on-Victorianshare-households.pdf]. 
Serna, H. M, Alzate, J., Ramírez, D. E. y Castro, E. S. (2019). La inserción laboral de los jóvenes en Colombia. Retos y perspectivas. Revista Jurídicas, 16(1), 42-61, [https://doi.org/10.17151/jurid.2019.16.1.4].

Viáfara, C. A. y Uribe, J. I. (2009). Duración del desempleo y canales de búsqueda de empleo en Colombia. Revista de Economía Institucional, 11(21), 139-160, [https://www.redalyc.org/articulo. oa?id=41911848009]. 